\title{
The Effect of Swelling Ratio on the Coulter Underestimation of Hydrogel Microsphere Diameters
}

\author{
Michael Pellegrini, BS, Abhimanyu Cherukupalli, BS, Michael Medini, BS, \\ Ron Falkowski, BS, and Ronke Olabisi, PhD
}

It has been demonstrated that the diameters of porous particles are underestimated by Coulter measurements. This phenomenon has also been observed in hydrogel particles, but not characterized. Since the Coulter principle uses the displacement of electrolyte to determine particle size, electrolyte contained within the swelled hydrogel microparticles results in an underestimate of actual particle diameters. The increased use of hydrogel microspheres in biomedical applications has led to the increased application of the Coulter principle to evaluate the size distribution of microparticles. A relationship between the swelling ratio of the particles and their reported Coulter diameters will permit calculation of the actual diameters of these particles. Using polyethylene glycol diacrylate hydrogel microspheres, we determined a correction factor that relates the polymer swelling ratio and the reported Coulter diameters to their actual size.

\section{Introduction}

$\mathbf{H}$ YDROGEL MICROPARTICLES ARE increasingly investigated for their use in biomedical applications. Their ability to swell many times their weight in an aqueous solution enhances nutrient, waste, or therapeutic molecule transport, making them ideal vehicles for drug delivery, cell encapsulation, and tissue engineering scaffolds. ${ }^{1}$ Hydrogel microspheres can be designed to degrade over time, permitting specified delivery rates of a therapeutic product. $^{2}$ When used to microencapsulate exogenous cells before transplantation, hydrogel microcapsules are an effective method to isolate these donor cells from the host immune response. ${ }^{3-5}$ When used as scaffolds for entrapped cells, hydrogel microparticles can be made in sizes that are within the diffusion distance of oxygen and can provide superior gas, nutrient, and waste exchange than larger hydrogel constructs. ${ }^{6}$ There are several methods to characterize the size distribution of these particles, predominantly through image processing and the use of particle analyzers that employ the Coulter principle. Since image processing can be timeconsuming, for many researchers an automated particle analyzer is the preferred method when analyzing microparticles. ${ }^{7-9}$

Particle analyzers using the Coulter principle send an electrolyte diluent through an aperture, in which a small electrical current is passed. An aspirated particle will have its own voltage drop, which alters the impedance measured at the aperture. This impedance change is directly proportional to the electrolyte volume displaced by the passing particles: for solid nonconducting particles, the displaced electrolyte volume is identical to the particle size; for porous nonconducting particles, the displaced electrolyte volume is less than the particle size due to retention of electrolyte within particle pores. ${ }^{10,11}$ Hydrogels swollen with electrolyte are highly permeable to ionic species when within an external electric field, which results in a much smaller impedance change, a lower calculated displaced volume, and hence, an underestimation of particle size. ${ }^{12}$ Others have observed this phenomenon with alginate microspheres, ${ }^{13}$ and we observed a similar size underestimation with our polyethylene glycol diacrylate (PEGDA) hydrogel microspheres and set out to determine a correction factor and relate it to hydrogel physical properties. This correction factor is relevant to multiple applications, since a variety of investigations have employed the Coulter method to estimate the size of their hydrogel microparticles. $^{14-24}$

\section{Materials and Methods}

\section{Microsphere formation}

Hydrogel precursor solutions were formed as previously described. ${ }^{4}$ Briefly, three aqueous precursor solutions were formed with the following molecular weight PEGDA: 5, 10, and $20 \mathrm{kDa}$. Each solution was prepared by combining the respective PEGDA $(10 \% \mathrm{w} / \mathrm{v})$ with triethanolamine $(1.5 \% \mathrm{v} / \mathrm{v})$,

Department of Biomedical Engineering, Rutgers University, Piscataway, New Jersey.

(C) M. Pellegrini et al. 2015; Published by Mary Ann Liebert, Inc. This Open Access article is distributed under the terms of the Creative Commons Attribution Noncommercial Licence (http//creativecommons.org/licences/by-nc/4.0) which permits any noncommercial use, distribution, and reproduction in any medium, provided the original authos(s) and the source are credited. 
Pluronic acid F68 (1.0\% v/v), $37 \mathrm{mM}$ 1-vinyl-2-pyrrolidinone, and $0.1 \mathrm{mM}$ eosin $\mathrm{Y}$ in HEPES-buffered saline (pH 7.4). A hydrophobic photoinitiator solution was produced by combining 2,2-dimethoy-2-phenyl acetophenone with 1-vinyl-2pyrrolidinone $(300 \mathrm{mg} / \mathrm{mL})$, which was in turn mixed with mineral oil $(3 \mu \mathrm{L} / \mathrm{mL})$. Microspheres were formed by combining the polymer precursor solution with the mineral oil solution $(200 \mu \mathrm{L} / \mathrm{mL})$ and generating a vortex-induced emulsion ( $2 \mathrm{~s}$ vortex) under white light $(20 \mathrm{~s}$ exposure) to photopolymerize the suspended droplets. Resulting microspheres were then isolated from the oil through centrifugation at $325 \mathrm{~g}$ for $5 \mathrm{~min}$. Following centrifugation, microspheres were filtered to concentrate their distribution to the $100-250 \mu \mathrm{m}$ range.

\section{Microsphere particle analysis}

Poly-L-lysine (MW: 68,600 g/mol; Sigma-Aldrich)coated alginate (MW: 100,000-200,000 kDa, G-content: 65-70\%; Sigma-Aldrich) microspheres received as a gift (the Yarmush Laboratory) and PEGDA microspheres $(5,10$, and $20 \mathrm{kDa}$ ) were imaged using phase contrast on an epifluorescent microscope (inverted Zeiss Axiovert). Images were processed using NIH ImageJ, which generated histogram size distributions of the filtered microspheres. Immediately following imaging, samples were collected and passed through a Coulter counter (Beckman Coulter Multisizer III fitted with a $1000 \mu \mathrm{m}$ aperture) to obtain histogram size distributions corresponding to those obtained through image processing; in short, each sample was analyzed twice to ensure that differences in resulting histograms were due to the methods and not the samples. The resulting histograms were compared.

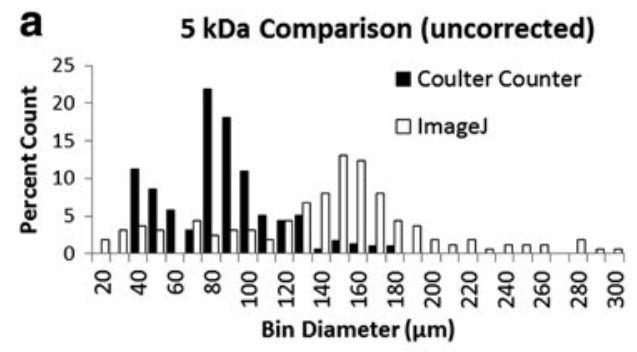

b $10 \mathrm{kDa}$ Comparison (uncorrected)

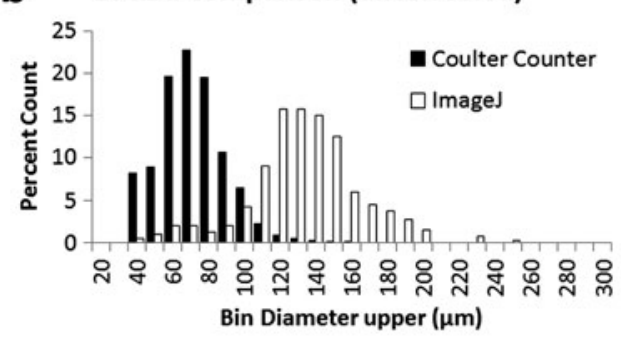

C $20 \mathrm{kDa}$ Comparison (uncorrected)

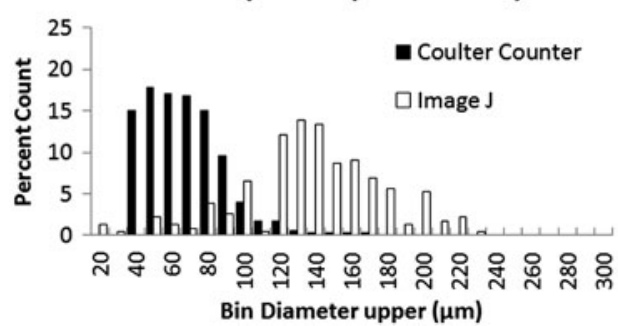

\section{Histogram comparisons}

Horák et al. described the underestimation of porous microparticle diameters by a factor $f$ given by the following:

$$
f=d_{\mathrm{c}} / d_{\mathrm{o}}=(1-p)^{1 / 3}
$$

where $d_{\mathrm{c}}$ and $d_{\mathrm{o}}$ are the Coulter and observed diameters, respectively, and $p$ is the porosity in terms of volume fraction of the electrolyte-filled pores. ${ }^{10}$ Since the porosity of solid porous particles does not correlate to the swelling of hydrogel microspheres, we sought to first determine a factor that would correct for the underestimation and then to relate the factor to a physical parameter of the hydrogel microspheres. Coulter and ImageJ histograms were input into a MATLAB code that compared the two histograms using the chi-square two sample test. The code numerically solved for $f$ by minimizing the chi-squared distance between the Coulter and ImageJ histograms.

\section{Hydrogel swelling ratio and mesh size calculation}

Precursor solution $(750 \mu \mathrm{L})$ containing $10 \% 5,10$, or $20 \mathrm{kDa}$ PEGDA was photopolymerized with white light in a glass mold to form 5-mm-thick hydrogel sheets measuring $\sim 25 \times 75 \mathrm{~mm}$. Disks $1 \mathrm{~cm}$ in diameter were punched from sheets, placed in phosphate-buffered saline (PBS) containing $0.05 \%$ azide, and allowed to swell for $24 \mathrm{~h}$ in a humidified incubator at $37^{\circ} \mathrm{C}$. The weight of each disk was recorded after swelling to equilibrium $\left(W_{\text {eq }}\right)$ and drying by lyophilization $\left(W_{\mathrm{dry}}\right)$ to determine the swelling ratios $(S)$, given by the following:

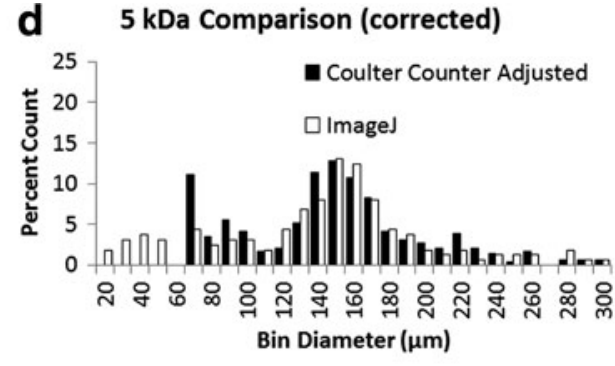

e $\quad 10 \mathrm{kDa}$ Comparison (corrected)

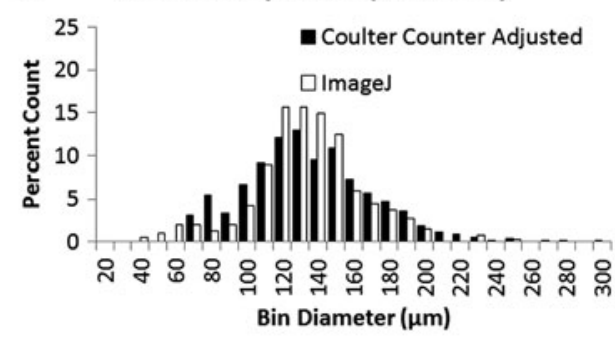

f $20 \mathrm{kDa}$ Comparison (corrected)

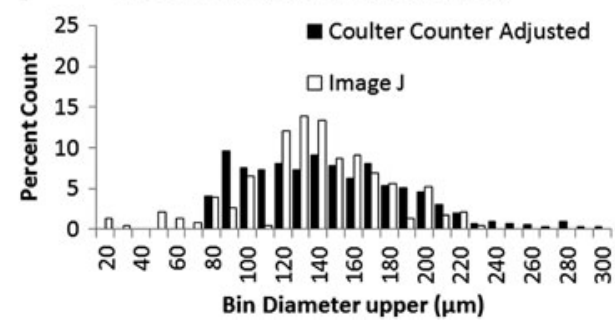

FIG. 1. Size distributions of polyethylene glycol diacrylate (PEGDA) hydrogel microspheres as measured by a Coulter counter and image analysis using NIH ImageJ. Identical samples are measured in each graph. (a-c) The distributions from each measurement are overlaid, with Coulter counts reporting smaller microspheres than measured by ImageJ. (d-f) After determining a correction factor, $f$, Coulter diameters were multiplied by $f$ and distributions were replotted with ImageJ results. 
Table 1. Hydrogel Physical Properties

\begin{tabular}{lcccc}
\hline Hydrogel & $\begin{array}{c}\text { Molecular } \\
\text { weight }(\mathrm{kDa})\end{array}$ & $\begin{array}{c}\text { Mesh size } \\
(\mathrm{nm})\end{array}$ & $\begin{array}{c}\text { Swelling } \\
\text { ratio }\end{array}$ & $\begin{array}{c}\text { Correction } \\
\text { factor }(\mathrm{f})\end{array}$ \\
\hline PEGDA & 5 & 16.2 & 14.5 & 0.553 \\
PEGDA & 10 & 28 & 14.6 & 0.507 \\
PEGDA & 20 & 32.3 & 16.5 & 0.434 \\
Alginate & $100-200$ & $\sim 10^{28}$ & $\sim 22.5^{29}$ & 0.125 \\
\hline
\end{tabular}

PEGDA, polyethylene glycol diacrylate.

$$
S=W_{\text {eq }} / W_{\text {dry }}
$$

Since it was not known whether polymer mesh size would also correlate to Coulter measurements, a dextran release study was performed as previously described to determine the mesh size of the various molecular weight hydrogels. ${ }^{25}$ Briefly, solutions of fluorescently labeled charge-neutral dextrans (5\% w/v; Sigma) were prepared by combining various dextran molecular weights $(10,20,40,70$, and $140 \mathrm{kDa}$ ) with the PBS-azide solution at $0.05 \mathrm{mg} / \mathrm{mL}$. The previously formed hydrogel disks were then swelled in $1 \mathrm{~mL}$ of each dextran molecular weight solution in a humidified incubator at $37^{\circ} \mathrm{C}$ to allow dextran diffusion into the gel. After $24 \mathrm{~h}$, the gels were removed from well plates, blotted dry, and placed in fresh PBS-azide solutions without dextran. Dextran effusion from the gels was measured after $24 \mathrm{~h}$ with fluorescent readings at ex/em 530/490. Fluorescent signals were converted to concentrations by comparison to dextran standard curves. Concentrations were first normalized by dividing values with gel weight, then normalized concentrations were plotted against published values for each molecular weight dextran hydrodynamic radius. ${ }^{26}$ The area $(A)$ under this curve is reported to give a quantitative measure of hydrogel permissivity for the range of hydrodynamic radii assayed. ${ }^{25}$ With the values for hydrogel permissivity, the relative mesh size can be determined by the following equation:

$$
\xi_{x} \sim\left(A_{x} / A_{\text {known }}\right)
$$

where $\xi_{x}$ is the relative mesh size of a hydrogel with molecular weight $x$ and permissivity $A_{x}{ }^{25}$ Since the relative mesh size for $10 \% 10 \mathrm{kDa}$ hydrogels formulated according to our methods has been determined to be $\sim 280 \AA{ }^{27}$ this value was used for $A_{\text {known }}$ to estimate the mesh sizes of the remaining hydrogels.

\section{Results}

Diameters measured by the Coulter counter were consistently smaller than those determined by imaging for every molecular weight PEGDA (Fig. 1a-c). Once a correction factor $(f)$ had been determined numerically (Table 1), Coulter distributions were multiplied by $f$ and overlaid with ImageJ distributions (Fig. 1d-f). Values for hydrogel swelling ratio, molecular weight, and mesh size were calculated to determine whether there was any correlation with $f$ (Table 1). For PEG hydrogels, the correction factor $f$ was quadratically related to hydrogel mesh size and linearly related to hydrogel molecular weight and swelling ratio. Alginate was used as a test material to evaluate whether the fits of $f$ could be used to predict values for $f$ in materials
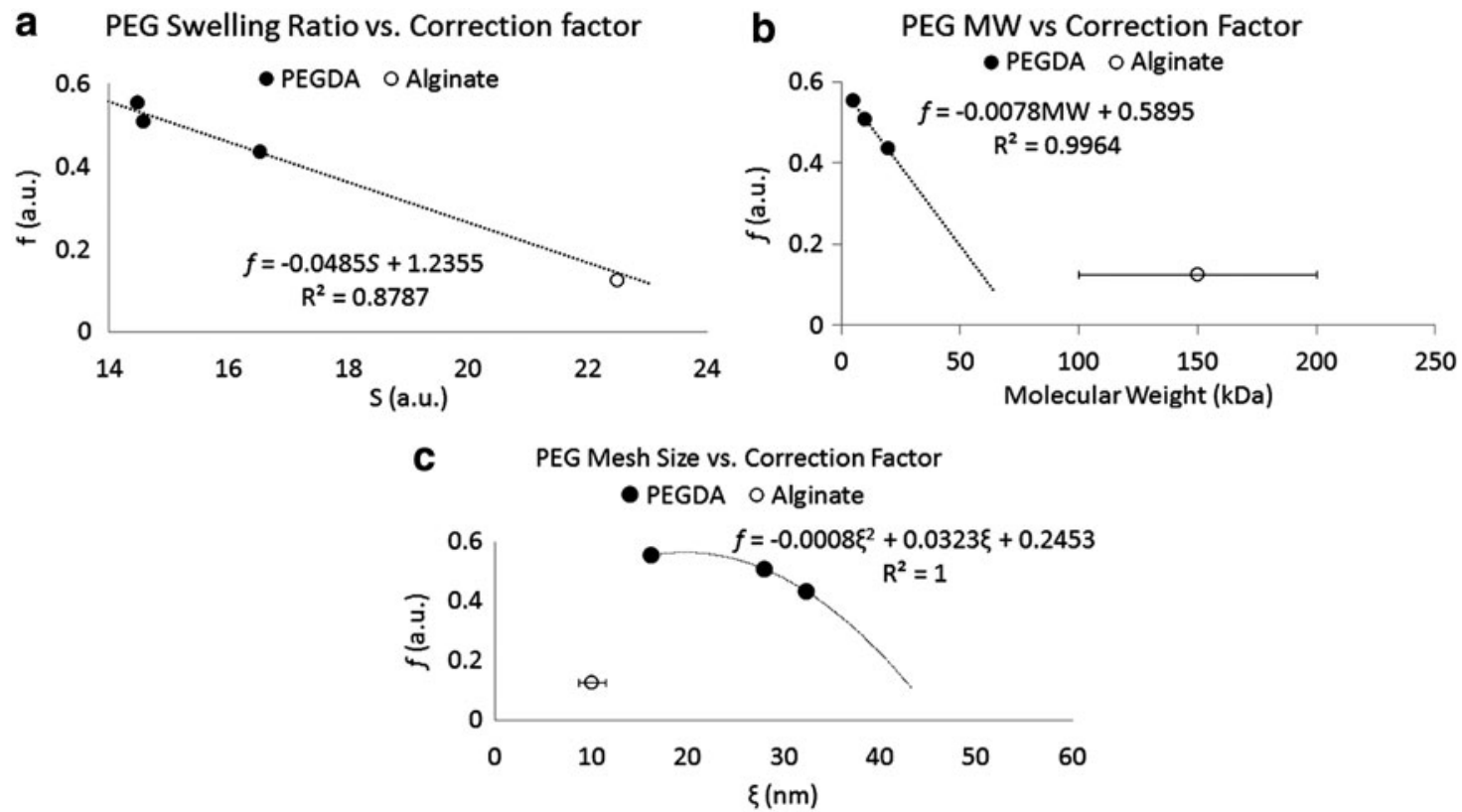

FIG. 2. Correction factor, $f$, plotted against hydrogel physical properties. Trendlines were fit only to PEGDA data, then alginate was plotted on the same graph to evaluate whether fit equations would predict alginate physical properties. (a) PEGDA hydrogel swelling ratios versus correction factor. Linear trendline equation closely predicts alginate values. (b) PEGDA hydrogel molecular weights versus correction factor. Linear trendline shows no relation to alginate molecular weights. (c) PEGDA hydrogel mesh size versus correction factor. Quadratic trendline shows no relation to alginate molecular weights. 
other than PEGDA. There was a correlation to the swelling ratio, but not to molecular weight or mesh size (Fig. 2), which predicted an $f$ of 0.144 compared to the actual numerically determined value of 0.125 . The empirical equation that relates the correction factor to the swelling ratio is as follows:

$$
f=-0.0485 S+1.2355
$$

\section{Discussion}

The results indicate that when using the Coulter principle to measure hydrogel microparticles, it is important to account for the effects of hydrogel swelling in electrolyte, which results in an apparent transparency of these microparticles to devices that use the Coulter principle. An $f$ correction factor of 1 would indicate that no correction was necessary and that Coulter and imaged microparticle measurements were identical. A correction factor $>1$ would indicate that Coulter measurements overestimated hydrogel microsphere sizes, while factors $<1$ indicate the underestimation of the microsphere size. Our results demonstrate decreasing $f$ correction values with an increasing swelling ratio, which show that as the hydrogels absorb more fluid the Coulter measurement becomes more inaccurate. As the particles swell more, greater amounts of electrolyte contribute to the volume of the microspheres, and hence, the Coulter measurements increasingly underestimate these particles. Equation (4) permits the correction of the Coulter measurements for PEGDA and alginate hydrogel microparticles. Future studies should evaluate Equation (4) against other molecular weight alginates and other hydrogel materials.

\section{Conclusions}

This correction factor has not been exhaustively evaluated against all of the different types of hydrogels that have been measured with the Coulter principle, which is a limitation of this study. However, the correction factor is important because it is applicable to both PEGDA and a common range of molecular weight alginates used to form microparticles. For alginates of different molecular weight, but with similar swell ratios, the correction factor should still be applicable. Since the majority of studies of microencapsulating mammalian cells use alginate, this correction factor is relevant to these studies. Coulter counting devices are convenient and less time consuming than image analysis; this correction factor enables researchers to continue to use their devices and obtain accurate measurements of their microparticles.

\section{Acknowledgments}

The first author was supported by a fellowship from the NASA sponsored NJ Space Grant Consortium. Alginate microspheres were made by Ileana Marrero Berríos of the Yarmush laboratory.

\section{Disclosure Statement}

No competing financial interests exist.

\section{References}

1. Fisher, S.A., Tam, R.Y., and Shoichet, M.S. Tissue mimetics: engineered hydrogel matrices provide biomimetic environments for cell growth. Tissue Eng Part A 20, 895, 2014.

2. Hoare, T.R., and Kohane, D.S. Hydrogels in drug delivery: progress and challenges. Polymer 49, 1993, 2008.

3. Acarregui, A., Herrán, E., Igartua, M., Blanco, F.J., Pedraz, J.L., Orive, G., and Hernandez, R.M. Multifunctional hydrogel-based scaffold for improving the functionality of encapsulated therapeutic cells and reducing inflammatory response. Acta Biomater 10, 4206, 2014.

4. Olabisi, R.M., Lazard, Z.W., Franco, C.L., Hall, M.A., Kwon, S.K., Sevick-Muraca, E.M., Hipp, J.A., Davis, A.R., Olmsted-Davis, E.A., and West, J.L. Hydrogel microsphere encapsulation of a cell-based gene therapy system increases cell survival of injected cells, transgene expression, and bone volume in a model of heterotopic ossification. Tissue Eng Part A 16, 3727, 2010.

5. Sun, A.M., Goosen, M.F., and O'shea, G. Microencapsulated cells as hormone delivery systems. Crit Rev Ther Drug Carrier Syst 4, 1, 1986.

6. Yeh, J., Ling, Y., Karp, J.M., Gantz, J., Chandawarkar, A., Eng, G., Blumling, J., 3rd, Langer, R., and Khademhosseini, A. Micromolding of shape-controlled, harvestable cell-laden hydrogels. Biomaterials 27, 5391, 2006.

7. Berkland, C., Kim, K.K., and Pack, D.W. Fabrication of PLG microspheres with precisely controlled and monodisperse size distributions. J Controlled Release 73, 59, 2001.

8. Wang, S., Zhao, W., Song, J., Cheng, S., and Fan, L. A platform for preparation of monodispersed fluorescent conjugated polymer microspheres with core-shell structures. Macromol Rapid Commun 34, 102, 2013.

9. Ambrose, C.G., Clyburn, T.A., Mika, J., Gogola, G.R., Kaplan, H.B., Wanger, A., and Mikos, A.G. Evaluation of antibiotic-impregnated microspheres for the prevention of implant-associated orthopaedic infections. J Bone Joint Surg Am 96, 128, 2014.

10. Horák, D., Peška, J., Švec, F., and Štamberg, J. The influence of porosity of discrete particles upon their apparent dimensions as measured by the Coulter principle. Powder Technol 31, 263, 1982.

11. van der Plaats, G., and Herps, H. A study on the sizing process of an instrument based on the electrical sensing zone principle. Part 2. The influence of particle porosity. Powder Technol 38, 73, 1984.

12. Ito, T., Sun, L., Bevan, M.A., and Crooks, R.M. Comparison of nanoparticle size and electrophoretic mobility measurements using a carbon-nanotube-based coulter counter, dynamic light scattering, transmission electron microscopy, and phase analysis light scattering. Langmuir 20, 6940, 2004.

13. Mukania, V., Stoppel, W., and Roberts, S. Investigation of the swelling and shrinkage behaviors of alginate capsules. University of Massachusetts REU Symposium, Amherst, MA, 2010.

14. Zhu, H., Srivastava, R., and McShane, M.J. Spontaneous loading of positively charged macromolecules into alginatetemplated polyelectrolyte multilayer microcapsules. Biomacromolecules 6, 2221, 2005.

15. Wands, I., Shepherd, D.E.T., and Hukins, D.W.L. Viscoelastic properties of composites of calcium alginate and hydroxyapatite. J Mater Sci Mater Med 19, 2417, 2008. 
16. Srivastava, R., Brown, J.Q., Zhu, H., and McShane, M.J. Stable encapsulation of active enzyme by application of multilayer nanofilm coatings to alginate microspheres. Macromol Biosci 5, 717, 2005.

17. Zhu, H., Srivastava, R., Brown, J.Q., and McShane, M.J. Combined physical and chemical immobilization of glucose oxidase in alginate microspheres improves stability of encapsulation and activity. Bioconjug Chem 16, 1451, 2005.

18. Serp, D., Mueller, M., Von Stockar, U., and Marison, I.W. Low temperature electron microscopy for the study of polysaccharide ultrastructures in hydrogels. II. Effect of temperature on the structure of $\mathrm{Ca} 2+$ alginate beads. Biotechnol Bioeng 79, 253, 2002.

19. Mladenovska, K., Cruaud, O., Richomme, P., Belamie, E., Raicki, R.S., Venier-Julienne, M.-C., Popovski, E., Benoit, J.-P., and Goracinova, K. 5-ASA loaded chitosanCa-alginate microparticles: preparation and physicochemical characterization. Int J Pharm 345, 59, 2007.

20. Baruch, L., Benny, O., Gilert, A., Ukobnik, M., Itzhak, O.B., and Machluf, M. Alginate-PLL cell encapsulation system Co-entrapping PLGA-microspheres for the continuous release of anti-inflammatory drugs. Biomed Microdevices 11, 1103, 2009.

21. Benchabane, S., Subirade, M., and Vandenberg, G.W. Production of BSA-loaded alginate microcapsules: influence of spray dryer parameters on the microcapsule characteristics and BSA release. J Microencapsul 24, 647, 2007.

22. Jay, S.M., and Saltzman, W.M. Controlled delivery of VEGF via modulation of alginate microparticle ionic crosslinking. J Controlled Release 134, 26, 2009.

23. Yoshida, M., Mata, J., and Babensee, J.E. Effect of poly (lactic co glycolic acid) contact on maturation of murine bone marrow derived dendritic cells. J Biomed Mater Res A 80, 7, 2007.

24. Bertram, J.P., Saluja, S.S., McKain, J., and Lavik, E.B. Sustained delivery of timolol maleate from poly (lactic-co- glycolic acid)/poly (lactic acid) microspheres for over 3 months. J Microencapsul 26, 18, 2009.

25. Jimenez-Vergara, A.C., Munoz-Pinto, D.J., BecerraBayona, S., Wang, B., Iacob, A., and Hahn, M.S. Influence of glycosaminoglycan identity on vocal fold fibroblast behavior. Acta Biomater 7, 3964, 2011.

26. Armstrong, J.K., Wenby, R.B., Meiselman, H.J., and Fisher, T.C. The hydrodynamic radii of macromolecules and their effect on red blood cell aggregation. Biophys J 87, 4259, 2004.

27. Liao, H., Munoz-Pinto, D., Qu, X., Hou, Y., and Grunlan, M.A. Influence of hydrogel mechanical properties and mesh size on vocal fold fibroblast extracellular matrix production and phenotype. Acta Biomater 4, 1161, 2008.

28. Eral, H.B., López-Mejías, V., O’Mahony, M., Trout, B.L., Myerson, A.S., and Doyle, P.S. Biocompatible alginate microgel particles as heteronucleants and encapsulating vehicles for hydrophilic and hydrophobic drugs. Crystal Growth Des 14, 2073, 2014.

29. Wang, M.S., Childs, R.F., and Chang, P.L. A novel method to enhance the stability of alginate-poly-L-lysine-alginate microcapsules. J Biomater Sci Polym Ed 16, 89, 2005.

Address correspondence to: Ronke Olabisi, PhD Department of Biomedical Engineering Rutgers University 599 Taylor Road Piscataway, NJ 08854

E-mail: ronke.olabisi@rutgers.edu

Received: May 28, 2015 Accepted: August 27, 2015 Online Publication Date: September 28, 2015 